\title{
Switching off the generator: technical and economic approach for storage based renewable energy systems for islands
}

\author{
E. Franzen, N. Strauch, C. Triebel, E. Bosch \& B. Richards \\ Younicos AG, Berlin, Germany
}

\begin{abstract}
While increasing the share of renewable energy to $20 \%$ is a challenge for grid stability, working towards a $100 \%$ renewable energy supply requires a completely new approach. Small island grids are the ideal platform to start achieving tomorrow's energy concepts today.

To develop the necessary technologies for a high level renewable energy island system, Younicos AG built a hardware and software development and test site in Berlin. The actual system testing shows that the ability to switch off the diesel generator - i.e. guarantee a stable grid exclusively using fluctuating sources and batteries and without rotating masses, gives the freedom to choose any renewable energy configuration between 0 and $100 \%$. Consequently, the renewable energy system layout can be set up according to the island's ecological and economical goals.

A hybrid system with a high renewable penetration can generate substantial savings by substituting large shares of fuel for electricity generation. Therefore, in addition to the technological solutions, procedures and business models are being developed to identify the economic optimal configuration for isolated systems under a given set of climate and load conditions. The technical approach, results of the test phase and key parameters of a supply system based on renewables and storage will be summarized in this paper.
\end{abstract}

Keywords: island, island energy system, renewable energy, renewable energy integration, battery storage, grid stability, system optimization. 


\section{Introduction}

Countries across the world have set the goal of increasing their share of renewable energy (RE) to $20 \%$ by 2020 and even higher in the longer term, to ensure their energy supply is sustainable. Taking this further, the energy supply in 2050 could be more than $50 \%$ renewable and thus the majority would be from RE. While increasing the share of renewable energy to $20-30 \%$ is a challenge for grid stability, pushing this share to $50 \%$ and beyond requires new approaches $[1,2]$.

Energy storage systems are required to lift this limit, to enable excess energy to be stored and used when renewable sources are non productive. Wind farms, solar parks and, increasingly, energy storage systems are available on the market. However, control solutions are required that allow stability in a grid based exclusively on decentralized, renewable generators and storage capacity in the megawatt range. Storage management, intelligent power electronics and the development of a top-level energy management system all play an important role.

While renewable energy shares up to $50 \%$ and beyond are a long term perspective for mainland grids, smaller grids, such as island networks reach this limit much more quickly. Installing just a few wind turbines or one solar park is often enough to reach the technical limits of the grid. At the same time, isolated grids often face the challenge of energy independence as their grids typically use a diesel generator as the key conventional electricity supply. The diesel needs to be imported at a high cost, which continuously increases the cost of electricity, but it also means that the islands are reliant on the delivery of oil. Establishing new energy concepts that will allow a departure from fossil fuels is therefore particularly interesting from an economical point of view for self-sufficient supply structures.

\section{Technical challenges in renewable island grid systems}

Grid stability is currently maintained by conventional power stations. They are required to operate at a minimum level (typically $30-50 \%$ of nominal capacity) to be able to provide the ancillary services required for grid stability.

The rotating mass of synchronous generators, used in conventional units such as diesel generators, acts as short-term storage in the grid, since the inertia absorbs all deviations from the operating point. The machine is speeded up or slowed down, depending on whether a step in the load is positive or negative. The resulting change in frequency is registered by the controllers in the power plant and is countered by adjusting the power, typically referred to as primary control.

In this way, the inertia and controls of the power plant act to stabilize the grid, before the centralized controls of a network adjust the set points and start or stop additional units. Furthermore, a generator is capable of briefly providing a current level that is several times its nominal output. This performance is usually the basis for grid protection. 
This means that these power plants are required to continue being operational, even if wind and solar energy could cover the electricity demand in a grid at a given point in time. As a result, wind and solar farms are powered down. This limits the share of the energy supply that can come from renewable sources.

If these power plants were now to be disconnected from the grid, the remaining units in the grid - renewable sources and storage systems - would have to take over these tasks in addition to generating and storing energy. Since both renewable sources and batteries are connected to the grid via inverters, the properties of the synchronous generators are no longer available to stabilize the grid. In grids with limited short circuit power, the behavior of the decentralized power plants in the event of a short circuit becomes particularly important.

In summary, the following requirements must be fulfilled by a new generation structure, in order to guarantee the supply security and power quality for island grids, as stipulated in EN 50160.

- Energy balance - the use of renewable energy sources should be maximized without risking the supply security

- Power balance - the power must be kept in balance, despite fluctuating generation, at all times through reliable cooperation between the components, in order to ensure grid stability

- Frequency and voltage must be regulated according to the requirements of the standards for power quality, in terms of the permissible tolerance band and quality requirements

- The participants should be connected via uniform communication standards, in order to enable the generation park to be extended without problems

- Sufficient short circuit power or alternative protection schemes must be available in the event of a short circuit, in order to activate existing circuit protection

\section{Technical approach to renewable island grid systems}

\subsection{Technical concept}

Storage plays a key role for achieving high renewable energy shares. If the geological formation allows, pumped hydro plants may be the storage system of choice. The approach described in the paper focuses on electro chemical batteries incl. inverter system, which can be applied independent of the location. The battery absorbs electricity whenever the renewable sources produce more than demanded by the customers and release electricity when the load exceeds the renewable generation. In this way, the energy balance can be met.

A control scheme has been implemented for the stable operation of renewable island grids without reliance on rotating generators. With regard to the control concept, a distinction is made between decentralized and centralized control requirements, as shown schematically in figure 1. 


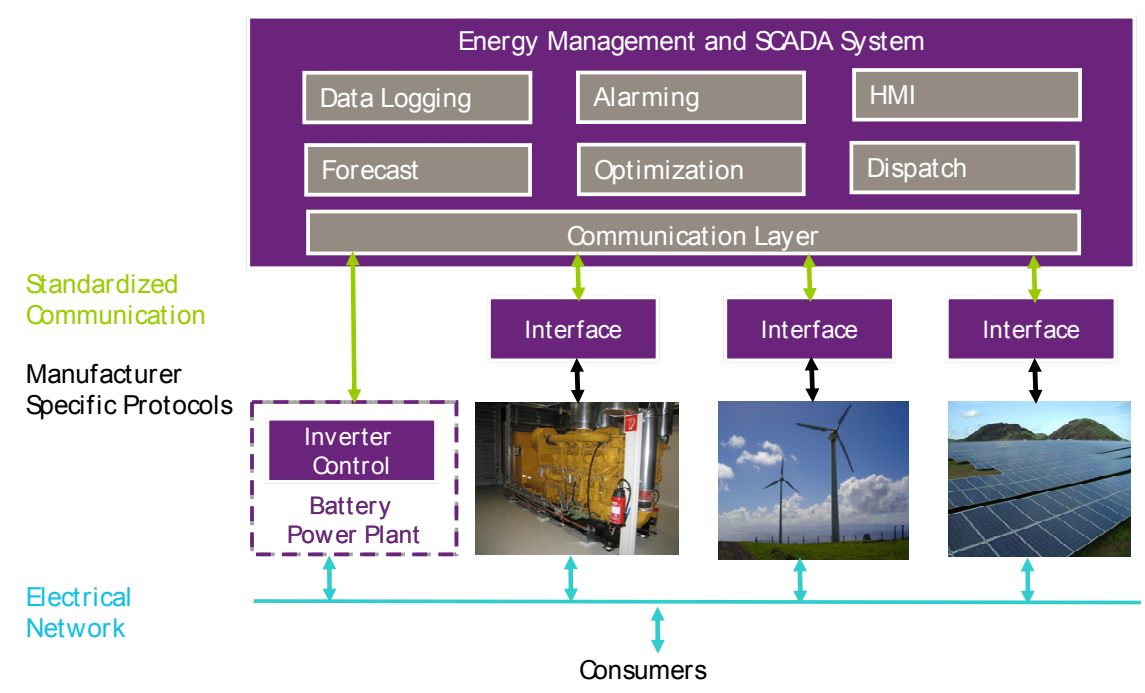

Figure 1: Concept for controlling island systems.

To decouple the availability of the grid from the availability of standard communications media, the components in the grid have sufficient intelligence to guarantee stable grid operation at all times. The control algorithms for the grid forming battery inverters enable the battery system to perform all required grid services including voltage and frequency control, black start, reserve power and provision of short circuit power. Being able to react in $\mu \mathrm{s}$, they ensure that the instantaneous power is balanced out, thus securing stability. This is achieved by combining intelligent and fast computing with well established control schemes in electricity networks. The interfaces to the production units allow for control and communication wise integration of both conventional and renewable plants.

As the central intelligence, the energy management is responsible for the optimal dispatch in the grid, based on forecasts for load as well as wind and PV power. It ensures that the energy is balanced, guaranteeing long-term supply security. Due to the capacity of the batteries, the processes controlled by the energy management are typically not critical in terms of time.

\subsection{Technology center}

To develop, optimize and demonstrate the necessary technology for a high level of renewable energy in an island system, a technology center has been built in Berlin. All power flows occurring in a self-sufficient supply grid can be simulated. The technology center as presented in figure 2 includes the components in an energy supply system and the equipment for implementing test scenarios based on load measurements and meteorological data from a specific location. The functions and performance data of the core components are described below. 

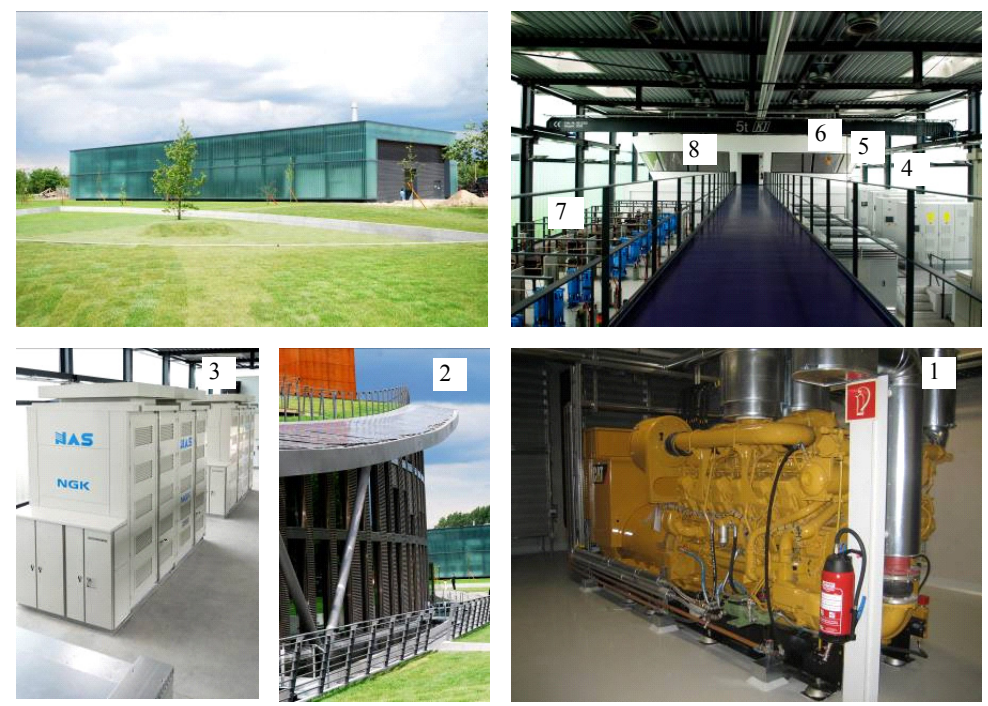

Figure 2: Technology center.

\section{(1) Diesel generator $-1 \mathrm{MW}$}

The diesel generator represents the conventional form of energy generation, as used currently in the majority of island grids that are not connected to the mainland by undersea cable.

\section{(2) Photovoltaic plant $-210 \mathrm{~kW}$}

The photovoltaic plant can be connected to the test bay to investigate the influence of its fluctuating supply on the whole system.

\section{(3) Sodium-sulfur (NaS) battery - 2x $500 \mathrm{kW/3} \mathrm{MWh}$}

The battery system consists of two separate battery blocks of $500 \mathrm{~kW} / 3 \mathrm{MWh}$ each plus battery inverters. The AC efficiency results in 75\% [3].

\section{(4) Source simulators $-2 \times 1 \mathrm{MW}$}

The dynamic response of wind turbines or photovoltaic plants can be simulated with the help of these inverters. The simulators use mathematical models of wind turbines and photovoltaic plants as input data, as well as high resolution climate data.

\section{(5) Load simulator - $1 \mathrm{MW}$}

This inverter serves to simulate the load response on an island. Measurements of consumption are used here for reference settings.

\section{(6) Auxiliary supply}

Depending on the mode of operation, energy can be drawn from or fed in to the public supply grid for the operation of the simulators. The island grid is decoupled from the main grid in terms of voltage and frequency via a DC coupling, giving the maximum flexibility for carrying out test trials. 


\section{(7) Medium voltage grid}

In grids with a connection power in the order of several MW the electricity is usually transported via a medium voltage distribution network. In order to take this into account, a $15 \mathrm{kV}$ medium voltage grid has been installed, including switchgear, transformers and a simulator for power lines $2-8 \mathrm{~km}$ long.

\section{(8) Control room}

The supervisory data acquisition and control system including HMI is located in the control room.

\section{(9) Short circuit switch}

A short circuit switch has been installed in the system, allowing specific short circuits and ground faults (one, two or three phase) to be generated in the medium voltage grid. With its help, protection concepts and the system response in the event of a fault can be analyzed and optimized.

\subsection{Test results}

The results of a test sequence carried out in the test bay - based on measurement data for the load, wind and irradiation - are detailed in the following section. Figure 3 shows the development of the frequency (top) and the power of all operating units (bottom).

The following units were used in the test: battery 1 (green), battery 2 (red), electrical load simulator (gray), wind simulator (light blue), photovoltaic simulator (blue) and the diesel generator (yellow). The frequency is controlled within the tolerance band from 49 to $51 \mathrm{~Hz}$ (EN 50160 for island grids) throughout the entire test.

A: At the start of the test (section A), the grid is formed by the two battery inverters. Subsequently, the load is connected. The batteries supply the load, charging the discharge power evenly.

B: Test the wind turbine, and photovoltaic simulators are connected. If the energy provided by the renewable sources is not sufficient to cover the load, the batteries will continue to be discharged. As soon as there is excess energy from the renewable sources, the batteries are charged.

$\mathrm{C}$ : The diesel generator is synchronized to the battery grid to show the parallel operation. The load sharing between the batteries and the diesel generator can be influenced by the respective controller settings. The diesel generator is switched off again without any disruption towards the end of section C.

D: In section D, both wind and PV are disconnected at once as it would be occur in case of a power line failure. Shortly before the point of the dropout of the renewable energy sources, the entire load (approx. $500 \mathrm{~kW}$ ) is supplied by the renewable energy sources and the batteries are being charged with around $500 \mathrm{~kW}$. The dropout of the renewable energy sources (due to the failure of a power line, for example) is compensated for instantaneously by the batteries. The batteries swap from charging at approx. $500 \mathrm{~kW}$ to discharging at $-500 \mathrm{~kW}$. 

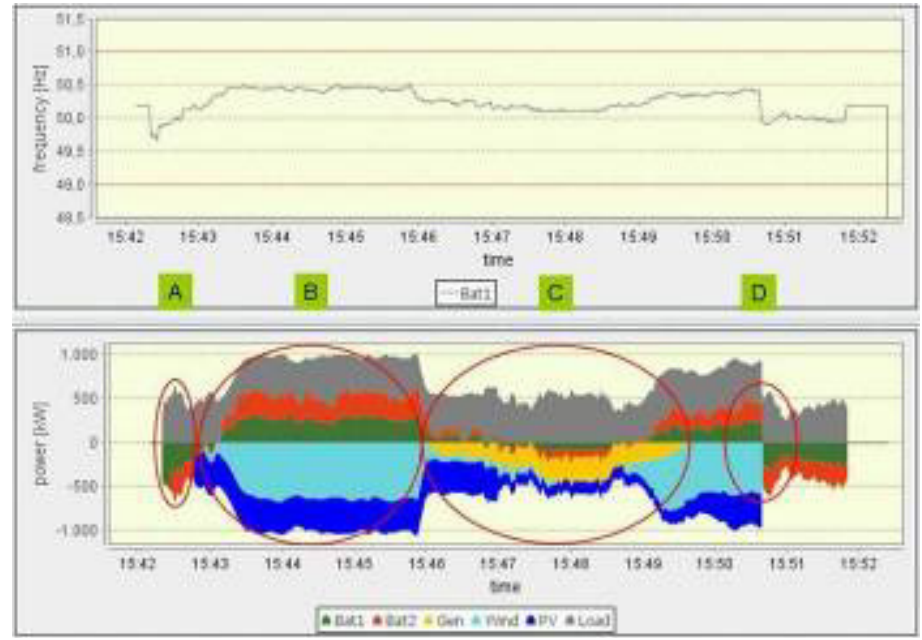

Figure 3: $\quad$ Power and frequency plots.

In a separate test, a 3-phase short circuit is intentionally created at the $15 \mathrm{kV}$ bus bar. The diesel generator, as well as the load and resource simulators, are disconnected for the purpose of this test. The short circuit voltages and currents, generated by the battery system, are documented in figure 4 . In this set up, the batteries supply a short circuit current of about 3.5 times their nominal current. The required short circuit capacity for a given project has to be chosen in accordance with the protection scheme.

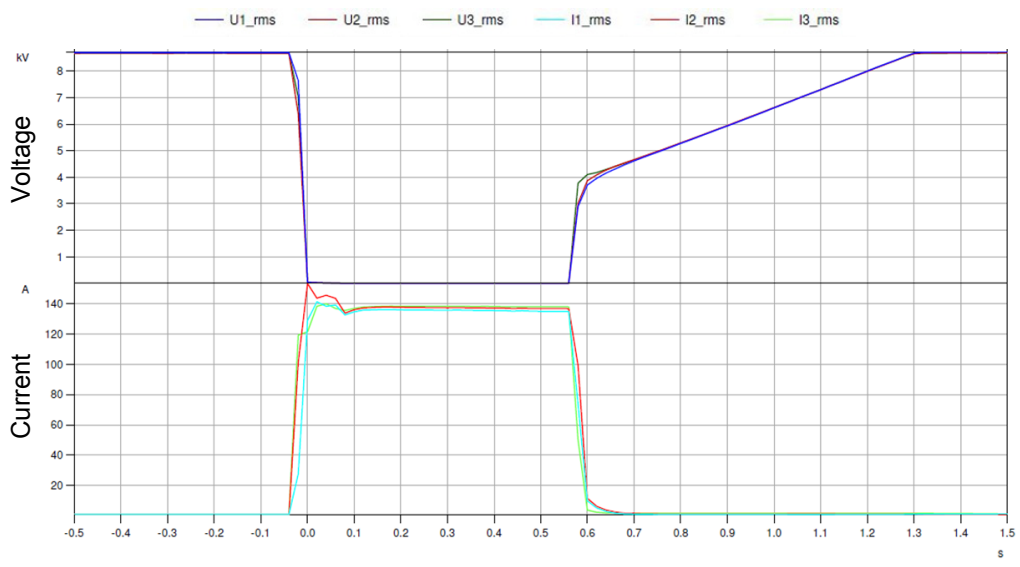

Figure 4: Voltages and currents during a short circuit. 


\section{Commercial aspects of renewable island grid systems}

Having ruled out the limitation of the renewable energy share due to technical constraints, the energy mix can then be chosen according to economic or environmental targets. Optimizing a renewable island grid project economically requires a set a different set of tools and analyses.

The sun and wind that comprise the fuel of island grids based primarily on renewable sources are both fluctuating and "free", uncovering a number of differences between the economics of traditional diesel based island systems and renewables based island systems. Primary among these differences are the following:

1. predominance of fixed, as opposed to variable, costs in the project economics

2. influence of site specific factors, particularly the cost of diesel delivered to the site, on the overall viability of the project economics

3. additional infrastructure requirements and potentially volatile asset utilization

All of these facts create a need for new compensation and financing schemes that go above and beyond the feed in tariffs currently understood by existing markets for renewable energy generation.

\subsection{Fixed vs. variable cost structures}

In the cost structure of traditional fossil fuel based electricity supply systems, the variable costs are typically predominant. These are usually comprised to a minor extend of the variable operation and maintenance costs and to a major extend of fuel acquisition costs. The installed capacity has to be sufficient to cover the peak demand taking all availability considerations into account. The amount of required fuel is then proportional to the energy consumption, the advantage being easy adaptability to varying energy needs. The disadvantage is, however, a strong vulnerability to volatile and, more often than not, increasing fuel prices.

Renewable energy and storage systems have - on the contrary - a cost structure which is mainly determined by capital and financing costs. Fuel, as mentioned above, is provided at no cost by the sun and wind. The technologies to convert this fuel into electricity and store it, however, must be purchased up front. When comparing the total system costs, then, one would have to compare the total cost of a renewable system to the cost of buying a diesel generator set and all of the diesel fuel for the next twenty years (or the relevant lifetime of the renewable energy system) on day one.

In sum, renewable systems allow for stable and predictable prices, however the associated project economics are more sensitive to variations in the load as the annual expenses are rather constant, irrespective of the actual output of the system. In addition, the initial investment is significantly higher compared to a fossil fuel based generation set up with the same annual production capability. Consequently, commercially viable renewable based island projects require new and different financing tools and vehicles. 


\subsection{Influence of site specific factors}

Optimizing the commercial viability of renewable based island grid projects requires a close analysis of the local conditions. Given the abovementioned fixed cost orientation of the economics of a renewable energy island system projects, their commercial viability increases up to, but not beyond, the point at which the specific production costs of the renewable hybrid system - including both renewable sources, storage and management system - reaches the variable cost of fuel based generation. Any installation of additional renewable energy assets beyond this point would then increase the overall specific cost of electricity. The fraction of the total load which can economically be substituted by renewables depends on several site factors, such as

- $\quad$ the magnitude (e.g. average wind speed and irradiation) and dynamics of renewable resources

- the time correlation of these resources with the load profile

- the modularity of the components in question and effects of scale

- the properties of the fuel based generators

- financial parameters such as the cost of capital, tax rates, inflation rates

- $\quad$ special local conditions such as logistic constraints or land availability

- the fuel price level

Especially the fuel price, which drives the variable costs of the fuel based generation, has a significant impact on the renewable energy share at which an overall economic optimum for the supply system is reached. Therefore, in addition to analysing the resources, technical and financial conditions at a given location, a reasonable assumption for the fuel price level is required to identify the most suitable overall system set up. Either actual fuel price levels or near term predictions or long term average fuel price predictions can be used to determine the optimal mix between fossil and renewable sources. Figure 5 schematically visualizes the impact of the fuel price on the break even level between fossil generation and renewable energy, which determines the optimum system configuration.

When developing commercially viable renewable based island grid projects, if the project structure and the modularity of the technical components allow, a ramp up of the renewable energy share along the actual fuel price levels is preferable as it reduces the risk of an inefficient investment. However, the possibility to reinvest at a later point may not be provided or the installation of certain equipment implies high offset costs (e.g. the transport of cranes for wind turbine installation to very remote locations). In these cases, it can be preferable to tailor the system to an expected long term average fuel price and to invest once for the project life time.

\subsection{Infrastructure asset requirements and utilization}

When replacing traditional fossil fuel generation with a large proportion of renewable energy generation sources, two effects can be observed which result in 
and increase of the specific costs per $\mathrm{kWh}$ for renewable energy - beyond the levels known for grid connected renewable energy plants today - as schematically indicated in figure 5.

1. Additional infrastructure, namely storage and more sophisticated energy management systems, is required to maintain security of supply and power quality.

2. The renewable plants will be powered down in times when the production potential exceeds the demand plus charging power or when the installed storage is fully charged. Consequently, the utilization factor of the renewable plants may be reduced. In case no storage is applied, this effect is worsened.

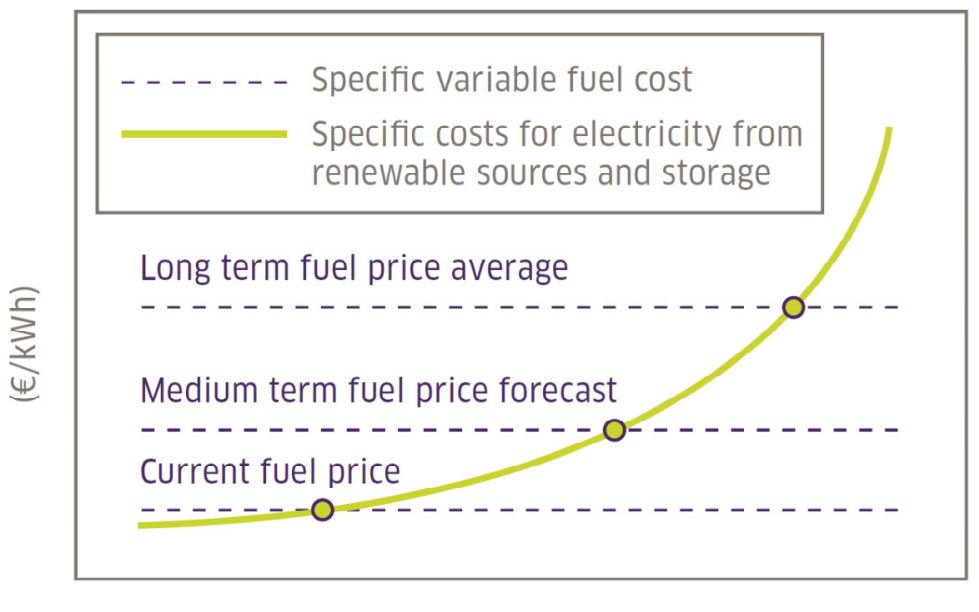

Renewable energy share (\%)

Figure 5: Renewable vs. fossil cost of electricity (schematic).

A commercially viable renewable energy island grid project must include increased revenue streams to cover the additionally required infrastructure, particularly the energy storage and energy management systems which must carefully be designed and integrated into the overall system so as to avoid under utilizing the renewable generation assets.

\subsection{Innovative compensation and financing schemes}

Traditional feed in tariffs for renewable energy sources grant priority feed in for renewable generation and allow for a fixed remuneration per $\mathrm{kWh}$ fed in during a project's life time in order to grant investment security. These tariffs were conceived in order to jump start the implementation and usage of renewable energy at a time when overall renewable energy penetration was low and component costs were high due to lack of needed scale. In high penetration renewable energy systems, the same tariffs are no longer applicable. 
New remuneration and financing schemes are being developed which take into account the fixed cost structure of these projects, the long term influence of site specific factors, particularly the development of the fuel price, and the required investment in additional infrastructure assets.

In case all assets of a renewable hybrid system are acquired by the institution responsible for the electricity supply and distribution, e.g. the local utility, the payback time and expected returns are the key parameters for the investment decision. In case an independent power producer (IPP) or a project company own the renewable generation plants and/or the storage system, suitable remuneration schemes have to be developed which balance the interest of the different parties: While the electric utility seeks to maximize the savings compared to the acquisition of fuel, the IPP or project company requires sufficient returns and investment security.

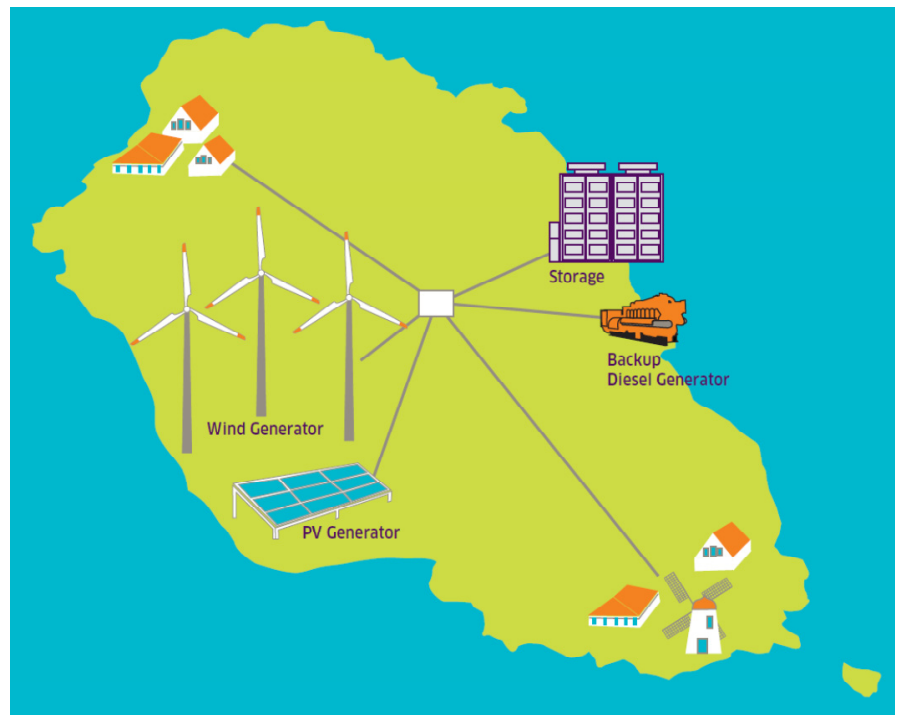

Figure 6: Graciosa Island.

\section{Pilot project Graciosa}

A pilot project is being developed on the island of Graciosa. With 4500 inhabitants and an area of $70 \mathrm{~km}^{2}$, Graciosa is one of the smaller islands of the Azores. The peak load is around $2.5 \mathrm{MW}$, with an annual electricity consumption of approx. $13 \mathrm{GWh}$.

Currently, around $85 \%$ of the electricity required is generated by diesel units; $15 \%$ is generated by an existing wind farm. The government of the Azores has set the political goal of providing the majority of the electricity supply with renewable energy sources by 2018. The energy provider in the Azores, EDA, has already implemented different renewable energy sources on the islands - from 
hydropower to geothermal to wind [4]. In cooperation with EDA, Graciosa was identified as a candidate to lead the fulfillment of the political goal by supplying the major share of the islands electricity needs from of wind, photovoltaic and batteries. A schematic overview is provided in figure 6 .

EDA continues to be the responsible entity for the generation, distribution and sales of electricity. A specially created project company owns and operates the renewable hybrid system. The energy management system of the project company interfaces with the diesel power station, e.g. to start and stop diesel engines depending on the state of charge of the battery, thus allowing for a system wide optimal dispatch. Younicos has the role of the project developer and turnkey supplier, including the layout, system simulation and system planning, as well as commercial and legal project development. The terms of the power purchase agreement with the utility have been agreed to and signed and construction is expected to begin in 2013, commissioning being scheduled for 2014.

\section{Conclusion}

The ability to switch off the diesel generator and to guarantee a stable grid exclusively using fluctuating sources and batteries and without rotating masses, gives the freedom to choose any configuration between 0 and 100\% renewables. Thus, the optimized system configuration can be set up according to the ecological and economical goals alone.

A hybrid system with a high proportion of renewable energy can generate substantial savings by substituting large shares of fossil fuel. Every island has specific characteristics in terms of climatic conditions, cost structures and financing options; therefore a careful analysis is required to determine the optimal economic and ecological system for a specific island.

With increasing costs for raw materials and a raised awareness about sustainable energy supplies, this kind of a decentralized supply concept is also relevant of regions within the national grid that wish to increase the amount of electricity generation coming from renewable resources.

\section{References}

[1] Brodersen, N.; Nabe, A.: Stromnetze 2020 plus, Gutachten im Auftrag der Bündnis 90/Grüne, 2009

[2] ETG Task Force Energiespeicher: Energiespeicher in Stromversorgungssystemen mit hohem Anteil erneuerbarer Energieträger, Verband der Elektrotechnik Elektronik Informationstechnik e.V., 2008

[3] Mizutani, T.: Innovative Technological Developments in Electricity Storage, Hannover Messe - World Energy Dialogue, 2009

[4] Amaral, R.: Azores Energy Work: A True Islenet, Islenet, Sustainable Energy Systems for European Islands Communities, Brussels 28-29 March 2006 